\section{Deutsche Mediziner verlassen die Schweiz}

Trotz höherer Löhne und eines besseren Arbeitsklimas kehren viele deutsche Mediziner der Schweiz mittlerweile den Rücken. Wie die Neue Zürcher Zeitung (NZZ) berichtete, entscheiden sich deutsche Ärzte immer häufiger aus Gründen des sozialen Umfelds gegen eine Anstellung in der Schweiz.

So berichtet im Online-Experten-Netzwerk coliquio eine Reihe von deutschen Ärzten von guten Erfahrungen im „Schlaraffenland“ Schweiz, im beruflichen wie privaten Umfeld. „Wir fühlen uns hier pudelwohl“, schreibt ein Mediziner, der mit seiner Frau und zwei Kindern seit 2012 in der Schweiz arbeitet. Andere Forumsteilnehmer warnen vor der Sprachbarriere und Ressentiments gegenüber Ausländern. Ein Zahnarzt erzählt, seine Frau sei im Job weggemobbt und die Kinder seien an der Schule als „blöde Nazis“ beschimpft worden.

Eine Ärztin meint in dem Forum: „Die Schweiz ist gut für Menschen ohne Kinder, Humor und Moral, die ist durch Geld ersetzt."

Dabei ist die Schweiz auf die Zuwanderung von medizinischem Fachpersonal angewiesen: Laut dem Schweizer Ärzteverband FMH haben 17,7 Prozent der in der Schweiz zugelassenen Ärzte einen deutschen Pass, das sind über 6.000 Personen, wie die NZZ schreibt. Außerdem stünden viele Hausärzte vor der Pensionierung, und die Zahl der nachrückenden Ärzte reiche nicht, um die Lücken zu füllen.

Hoffnung kommt für die Schweiz aus einem anderen Nachbarland: Für französische Fachkräfte ist laut NZZ die Attraktivität des Schweizer Arbeitsmarkts anscheinend ungebrochen.

\section{$m$}

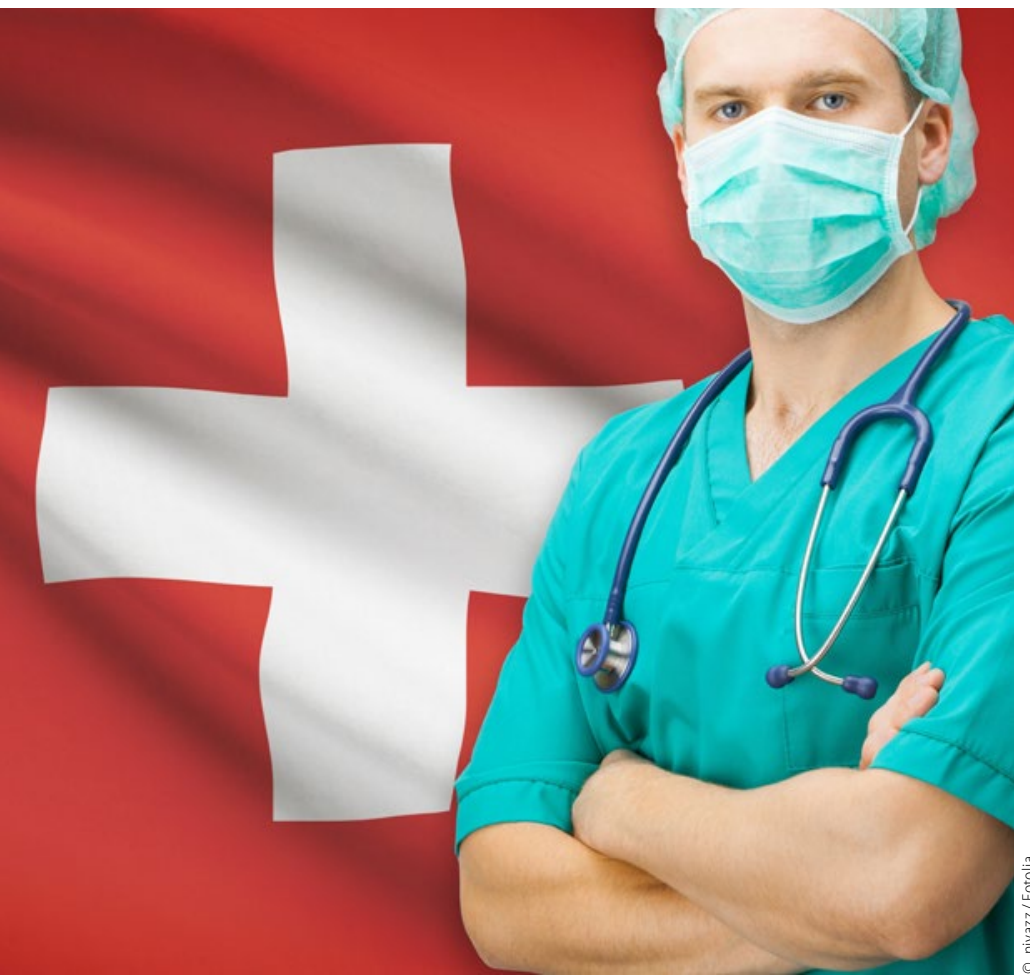

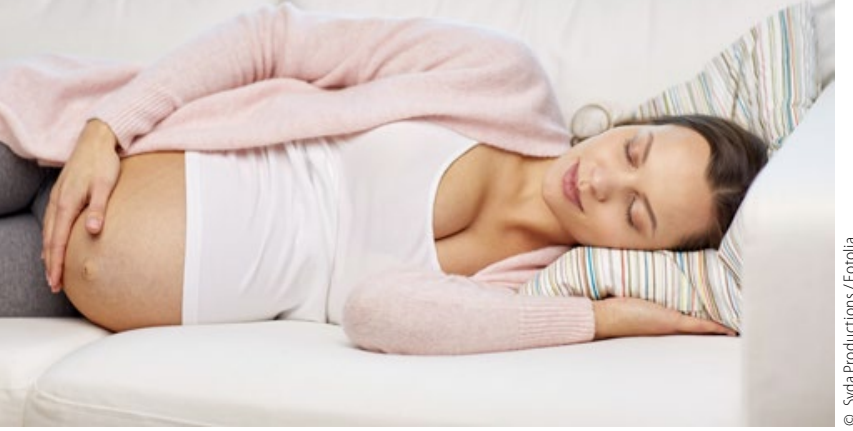

Neuerung für werdende Mütter

\section{Mutterschaftsgeld für selbstständig tätige Frauen}

Selbstständige privat krankenversicherte Frauen haben künftig die Möglichkeit, Mutterschaftsleistungen in Höhe des Krankengeldes zu erhalten. Für diese Neuerung hat der Bundestag den Weg frei gemacht, wie die CDU/CSU-Bundestagsfraktion mitteilte. Mit der Änderung des Versicherungsvertragsgesetzes werden die privaten Krankenversicherer dazu verpflichtet, eine Krankentagegeldversicherung anzubieten. Dadurch können sich selbstständig Privatversicherte in den letzten Wochen vor und den ersten Wochen nach der Entbindung absichern. „Bislang haben viele junge Frauen vor der Familienphase den Schritt in die Selbstständigkeit auch deswegen gescheut, weil es ihnen in der Anfangsphase finanziell nicht möglich gewesen wäre, aus eigenen Mitteln eine Auszeit aus ihrer beruflichen Tätigkeit zu finanzieren. Dieses Hindernis haben wir aus dem Weg geräumt", erklärten die stellvertretende Vorsitzende der Unionsfraktion im Bundestag, Nadine Schön, und die gesundheitspolitische Sprecherin der CDU/CSU, Maria Michalk, in einer gemeinsamen Pressemitteilung.

cas

\section{Maßnahmen gegen Desinfektionsmuffel}

Offenbar nehmen es viele Krankenhausärzte gerade bei der Präventionsmaßnahme Nummer 1, der Händedesinfektion, nicht so genau, wie die Ärzte Zeitung nun berichtet.

Demnach seien laut einer Studie des Medizinpsychologen Dr. Thomas von Lengerke von der Medizinischen Hochschule Hannover zwischen 2012 und 2013 die Compliance-Raten bei der Händedesinfektion im Rahmen der Aktion „Saubere Hände" unter anderem an Intensivstationen der Hochschule Hannover nach Anfangserfolgen wieder auf das Ausgangsniveau zurückgefallen (Ärzte: 48 Prozent, Pflegende: 56 Prozent).

Bei einer Kontrollgruppe sei die Einhaltung der Hygienerichtlinien im Jahr 2015 auf 70 Prozent gestiegen, allerdings zeigte sich hier der Erfolg vor allem bei den Pflegenden. Besonders niedrig sei die Desinfektionsquote bei den Ärzten sogar vor aseptischen Tätigkeiten, etwa vor dem Katheterlegen, gewesen. „Kein Motivationsproblem, sondern ein Umsetzungsproblem“, so von Lengerke. Sein Lösungsvorschlag: „Compliance-Raten und Infektions-Raten übereinander zu legen, um die Effekte sichtbar zu machen." Außerdem plädiert von Lengerke für Teamschulungen auf den Stationen, um die Compliance auch bei Ärzten zu steigern.

Auch führungsorientierte Maßnahmen könnten im hierarchischen Krankenhaussystem die Hygienemaßnahmen verbessern. $m r$ 